\title{
A laboratory comparison of four positive pressure ventilators used in the home
}

\author{
I.E. Smith, J.M. Shneerson
}

A laboratory comparison of four positive pressure ventilators used in the home. I.E. Smith, J.M. Shneerson. CERS Journals Ltd 1996.

ABSTRACT: Four brands of ventilator used for noninvasive positive pressure ventilation in the home were assessed to determine their performance on a patient simulator.

We tested the tidal volume ( $V T)$ preset Companion 2801 (Puritan Bennett), minute volume preset Monnal D (Taema), and two pressure preset ventilators, the Nippy (Friday Medical) and the Bilevel Positive Airway Pressure (BiPAP) (Respironics). A patient simulator was employed to investigate the relationships between $V \mathrm{~T}$, peak airway pressure (PAP) and mean airway pressure (MAP), the responses to an additional leak in the circuit and patient effort of a variable duration, which was modelled using a negative pressure pump.

For equivalent $V$ Ts, $>300 \mathrm{~mL}$, the Monnal $\mathrm{D}$ and Nippy generated a PAP up to $100 \%$ greater than the Companion 2801 and BiPAP. When an additional leak was introduced to the circuit, the $V T$ of the Companion 2801 and Monnal D fell by $>50 \%$, while the Nippy and the BiPAP responded by increasing flow and maintained $V$ T close to the level achieved with no leak. When the ventilators were triggered by a simulated patient effort of $0.25 \mathrm{~s}$ duration with a frequency $33 \%$ greater than that of the ventilator, the minute volume increased by $41 \%$ for the Companion 2801, by $18 \%$ for the Monnal D (no change expected), and by $17 \%$ for the Nippy (less than expected), and fell by $7 \%$ for the BiPAP due to irregular triggering. When patient effort was prolonged to $1.5 \mathrm{~s}$, a similar length to the inspiratory time of the ventilators, there was no further change in the minute volume of the Companion 2801 and Monnal D, while that of the Nippy and of the BiPAP increased by 38 and $71 \%$, respectively, compared to baseline.

These results show that distinct brands of ventilator respond to changes in the patient and patient circuit in different ways, which are not always predictable from a simple description of their operating principles. This should be borne in mind when choosing a positive pressure ventilator for noninvasive ventilation.

Eur Respir J., 1996, 9, 2410-2415.

The Respiratory Support and Sleep Centre, Papworth Hospital, Cambridge, UK.

Correspondence: I.E Smith

The Respiratory Support and Sleep Centre Papworth Hospital

Papworth Everard

Cambridge CB3 8RE

UK

Keywords: Home mechanical ventilation mechanical ventilators

positive pressure ventilation

\section{Abbreviations}

BiPAP=Bilevel Positive Airway Pressure $\mathrm{PAP}=$ peak airway pressure $\mathrm{MAP}=$ mean airway pressure

Received: November 231995

Accepted after revision August 111996
Noninvasive intermittent positive pressure ventilation (NiIPPV) is established as a safe and effective form of ventilatory assistance in subjects with neuromuscular or chest wall disorders $[1,2]$. It has been shown to be effective in providing long-term support in the home [3, 4]; to be an alternative to intubation for acute on chronic respiratory failure [5], and a means of weaning patients from invasive ventilation when conventional approaches have failed $[6,7]$. Its role in the long-term support of patients with chronic obstructive pulmonary disease (COPD) has yet to be clearly established but some positive results have been published [4, 8]. As experience with the techniques of NiIPPV has grown, certain limitations have become apparent. Therefore, interest in the details of the interactions between the patient and ventilator is increasing [9].

The positive pressure pumps currently available support ventilation using various methods. Theoretical models of some of these have been examined [10], and there have been some clinical studies comparing different ventilators [11-13]. However, to our knowledge, there are few published data comparing the bench performance of different ventilators used for NiIPPV. This is essential if meaningful comparisons are to be made between machines and if simple techniques, such as monitoring mask pressure, are to be used in the diagnosis of failures of the patient/ventilator interaction [14]. We therefore assessed four ventilators with different operating principles on a patient simulator to determine the relationships between tidal volume $(V \mathrm{~T})$, peak airway pressure (PAP) and mean airway pressure (MAP), the response to an additional leak in the circuit, and patient effort of varying duration.

\section{Methods}

\section{Ventilators examined}

The four brands of ventilators investigated are all currently used at home by patients of the Respiratory Support 
Centre (RSC), Papworth Hospital. They were chosen to exemplify different methods of supporting ventilation.

Companion 2801 (Puritan Bennett Ltd). The tidal volume $(V \mathrm{~T})$ and minimum respiratory frequency $\left(f^{\prime} \mathrm{R}\right)$ are preset. The inspiratory time $(t \mathrm{I})$ is preset by adjusting the inspiratory flow rate. Control, assist/control and synchronized intermittent mandatory ventilation without pressure support modes are offered. In the assist/control mode, the $f^{\prime} \mathrm{R}$ is preset and inspiratory flow rate (and hence $t \mathrm{I}$ ) remains fixed, but the patient can shorten the expiratory time $(t \mathrm{E})$ through pressure-triggering the transition to inspiration.

Monnal D (Taema Ltd). The minute volume is preset and $V \mathrm{~T}$ depends upon $f^{\prime} \mathrm{R}$. It operates in an assist/control mode with pressure-triggering from expiration to inspiration. It differs from the other ventilators in that it has a reservoir bag, which fills during expiration and supplements flow particularly during early inspiration.

Nippy (Friday Medical Ltd). The Nippy is a pressure preset ventilator. The $t \mathrm{I}$ is preset along with the maximum $t$ E. It operates in an assist/control mode with pressure-triggering from expiration to inspiration. The threshold negative pressure for triggering is preset and is three times more negative in the first half of the maximum expiratory time compared to the second half, with the aim of preventing breath-stacking.

The Bilevel Positive Airway Pressure-spontaneous and timed (BiPAP-ST; Respironics Ltd). This is a pressure preset device which was initially developed as an alternative to continuous positive airway pressure for the treatment of obstructive sleep apnoea [15]. The pressure in the patient circuit alternates between two levels, inspiratory and expiratory positive airway pressure (IPAP and EPAP, respectively), which can be independently adjusted. In the "timed" mode, $f^{\prime} \mathrm{R}$ and the ratio of $t$ I to $t \mathrm{E}$ (I:E ratio) are preset. In the "spontaneous" mode, there is no minimum $f^{\prime} \mathrm{R}$ and the transitions from inspiration to expiration and from expiration to inspiration are both triggered by flow criteria rather than by pressure thresholds. In the "ST" mode, the minimum $f^{\prime} \mathrm{R}$ is preset but both $t \mathrm{I}$ and $t \mathrm{E}$ may alter according to flow criteria.

The circuits for the Companion 2801, Monnal D and Nippy all contain an expiratory valve, which is closed during inspiration by the inflation of a balloon. The BiPAP-ST has a "whisper" valve, which acts as a fixed resistance leak in the circuit and is open throughout the respiratory cycle.

\section{Experimental set-up}

All measurements were made using a patient simulator (fig. 1), consisting of a bellows type lung simulator (Ohmeda) with adjustable resistance and elastance contained within a box connected to a negative pressure cuirass pump (Negavent; Dima Italia). The elastance and the resistance of the lung simulator were set to 20 $\mathrm{cmH}_{2} \mathrm{O} \cdot \mathrm{L}^{-1}$ and $5 \mathrm{cmH}_{2} \mathrm{O} \cdot \mathrm{L}^{-1} \cdot \mathrm{s}$, respectively. The ventilators were connected to the lung simulator using the

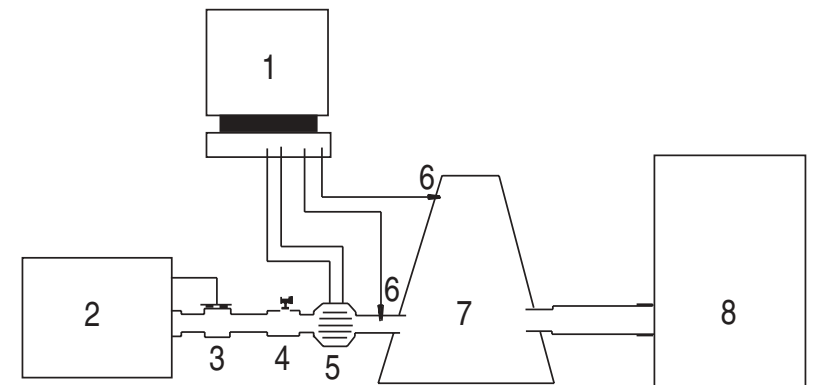

Fig. 1. - Experimental set-up. 1) Data logger; 2) positive pressure ventilator; 3) expiratory valve; 4) variable leak; 5) pneumotachograph; 6) pressure transducer; 7) lung simulator within a box; 8) negative pressure pump.

circuits provided by the manufacturers (elastance $>1,000$ $\mathrm{cmH}_{2} \mathrm{O} \cdot \mathrm{L}^{-1}$ in each case). A connector with a leak of radius $2 \mathrm{~mm}$, which could be opened or closed, and a pneumotachograph (Si-Plan Electronics Research) were placed between the expiratory valve (or whisper valve) and the lung simulator. The box was not airtight but had a leak sufficient to allow the expansion of the bellows, with no measurable rise in pressure within the box. Patient effort was simulated using the negative pressure cuirass pump. The accuracy and precision of this pump have previously been investigated and found to be better than $5 \%$ for $t \mathrm{I}$ and pressure within the hose, despite a leak into the box comparable to that in this model [16].

Pressure within the patient circuit, between the pneumotachograph and the lung simulator, and within the box were measured using calibrated pressure transducers (Vyggo). The inspiratory flow signal from the pneumotachograph was integrated to give a volume signal and was calibrated using a $1 \mathrm{~L}$ syringe (Vitalograph). All signals were sampled at $33 \mathrm{~Hz}$ and recorded digitally on a data-logging system (Cardas). When any variable, such as leak, was changed, 10 breaths were allowed for the system to stabilize and measures of PAP in the patient circuit, inspiratory $V \mathrm{~T}, f^{\prime} \mathrm{R}$ and $t \mathrm{I}$ were recorded and averaged for the next 20 breaths. MAP was calculated for the last three breaths by averaging the pressure levels at intervals of $0.1 \mathrm{~s}$ from the start of the upstroke until the pressure returned to baseline. The ventilators were tested in assist/control (ST for BiPAP) mode with a $f^{\prime} \mathrm{R}$ of 15 breaths $\cdot \mathrm{min}^{-1}$ and $\mathrm{I}: \mathrm{E}$ ratio of $1: 2$ (not adjustable on BiPAP). No pressure limit was set on the volume preset ventilators (Companion 2801 and Monnal D). On the BiPAP, EPAP was set to the minimum level $\left(2 \mathrm{cmH}_{2} \mathrm{O}\right)$ throughout.

\section{Investigations performed}

The relationship between $\mathrm{V} T, P A P$ and MAP. The preset pressure of the Nippy and BiPAP and the preset volume for the Companion 2801 and Monnal D were varied through a range, and the resulting $V \mathrm{~T}$ and PAP, respectively, were recorded. The results were plotted as a graph of $V \mathrm{~T}$ against PAP and related to the pressure waveforms of the individual ventilators. The ratio of PAP to MAP was calculated for each ventilator across the range of VT. Differences between the means of these ratios were compared for the different ventilators using unpaired t-tests. 
The effect of an additional leak in the patient circuit. For each ventilator, the $V \mathrm{~T}, \mathrm{PAP}$ and $t \mathrm{I}$ were measured with the leak between the expiratory valve and the pneumotachograph closed and then open, over a range of preset volumes (Companion 2801 and Monnal D) or preset pressures (Nippy and BiPAP).

The effect of patient effort. The ventilators were set up to produce a $V \mathrm{~T}$ of $550 \mathrm{~mL}$. The negative pressure pump was set to generate a pressure of $-8 \mathrm{cmH}_{2} \mathrm{O}$ within the box 20 times per minute. The negative pressure in the box models negative intrapleural pressure. Expansion of the lung simulator produced flow and negative pressure in the ventilator circuit sufficient to trigger the positive pressure ventilators. The I:E ratio of the negative pressure pump was varied such that the duration of the negative pressure within the box ranged $0.25-2 \mathrm{~s}$. On this test, the BiPAP demonstrated cyclical failures of triggering with cycle durations of up to $18 \mathrm{~s}$. Since it alters its triggering criteria over time, the BiPAP was run under these conditions for at least $10 \mathrm{~min}$ to discover whether the patterns of triggering were stable.

For each ventilator under any single test condition the ranges of $V \mathrm{~T}$, PAP and $t \mathrm{I}$ were $< \pm 5 \%$ of the mean, with correspondingly smaller SDS. Differences between test conditions and between ventilators were considerably larger and so no statistical tests were employed on these measures.

\section{Results and discussion}

\section{Relationship between $\mathrm{VT}$ and PAP}

The relationship between $V \mathrm{~T}$ and PAP for each of the four ventilators is shown in figure 2 . It can be seen that for a comparable $V \mathrm{~T}$, of greater than $300 \mathrm{~mL}$, the PAPs of the Monnal D and the Nippy were consistently higher than those of the Companion 2801 and the BiPAP. These differences can be related to the pressure waveforms of the different ventilators, which are reproduced in figure 3.

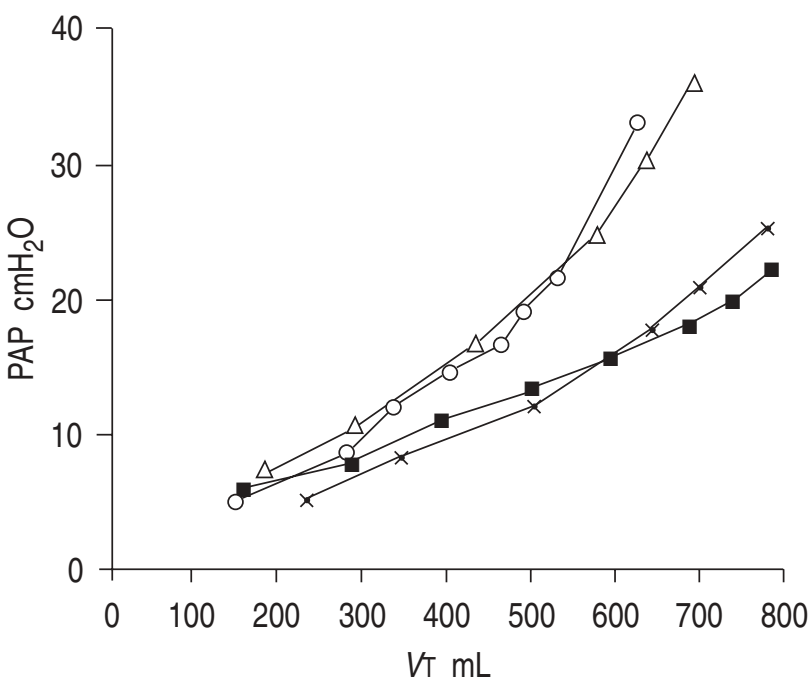

Fig. 2. - The relationship between peak airway pressure (PAP) and tidal volume $(V \mathrm{~T})$ for four distinct brands of ventilator. Monnal D (Taema); $\longrightarrow$ - Nippy (Friday Medical); $\longrightarrow$ : Companion 2801 (Puritan Bennett); —— : Bilevel Positive Airway Pressure (BiPAP; Respironics).
The initial peak of the Monnal D is produced by the reservoir bag supplementing flow early in inspiration. This was confirmed by removing the bag (fig. 3 ). As a consequence of these waveforms, the MAP of the different ventilators represented a different proportion of PAP. Over the range tested, the means (SD) of the MAP to PAP ratio were: $\mathrm{BiPAP} 0.79$ (0.03); Companion 2801 0.73 (0.08); Nippy 0.61 (0.02); and Monnal D 0.58 (0.09). Using unpaired t-tests, there was no significant difference between the MAP to PAP ratio for the BiPAP and the Companion 2801, but these were significantly higher $(\mathrm{p}<0.01)$ than those for the Nippy and the Monnal D.

The pressure waveform of the ventilator is thought to be important to patient comfort and the ease with which the patient becomes accustomed to the treatment [11, 17]. We have shown that the shape of the pressure waveform can have a major impact on the relationship between $V T$ and PAP. The Companion 2801 and BiPAP ventilators produced higher MAP to PAP ratios under the test conditions employed, compared to the Monnal D and the Nippy, which produced peaks of pressure most marked at the beginning and end of inspiration, respectively. As
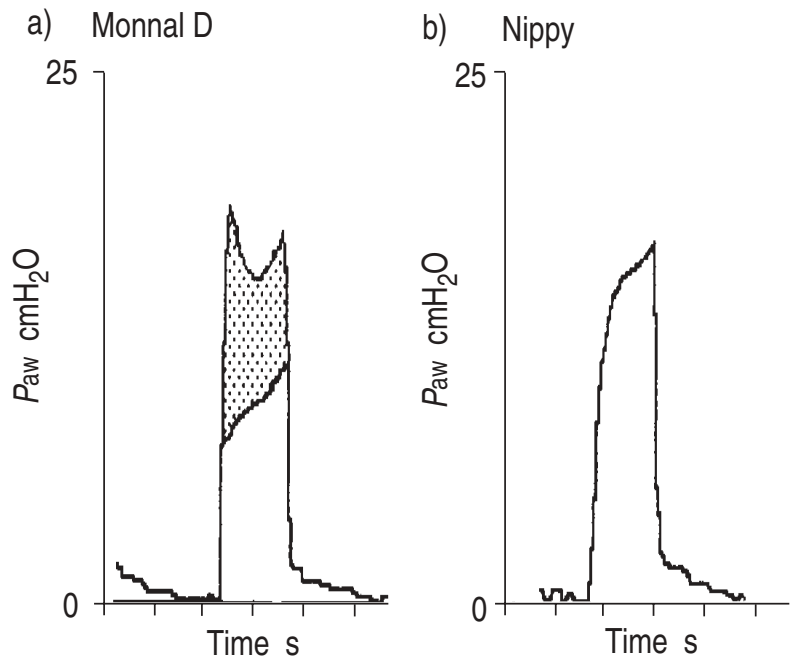

c) Companion 2801
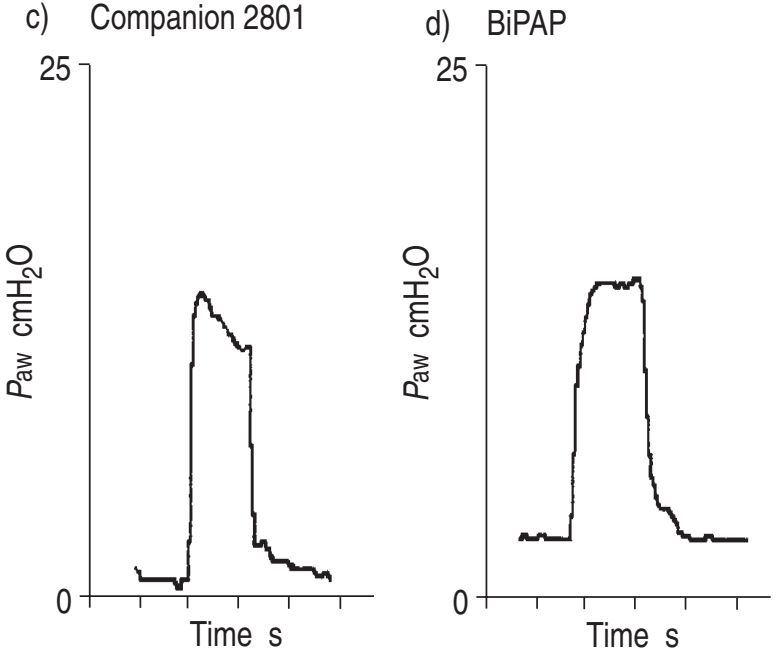

Fig. 3. - Airway pressure $(P$ aw $)$ waveforms of the four ventilators: a) Monnal D (Taema); b) Nippy (Friday Medical); c) Companion 2801 (Puritan Bennett); d) Bilevel Positive Airway Pressure (BiPAP; Respironics). The waveform of the Monnal D is drawn as a composite showing the contributions of the compressor (plain) and the reservoir bag (shaded). 
a consequence, the PAP for the latter two ventilators may be up to $100 \%$ greater than that for the former two ventilators for a given $V \mathrm{~T}$. This phenomenon has been demonstrated in awake patients, where the degree of ventilatory support was similar using the BiPAP and the Nippy, when the PAP of the former was a mean of 5.75 $\mathrm{cmH}_{2} \mathrm{O}$ lower $(\mathrm{p}=0.001)$ than that of the latter [11], although the authors do not remark upon this finding.

The most frequent complications of NiIPPV are pressure related. Aerophagia may lead to patient discomfort. Pressure sores produced by nasal or full face masks are more likely if the mask is tightly applied and the tension in the straps required to maintain a seal depends on the airway pressure. Barotrauma to the lung due to raised inspiratory pressures has rarely been reported [18], but in a population with compromised respiratory function would be a serious complication and the risk should be minimized where possible. Whether a high PAP or high MAP is more likely to cause any of these complications is not known, and further investigation is required before recommendations can be made on the choice of a ventilator based on its pressure waveform.

A knowledge of the pressure waveforms of the ventilator under standard conditions allows some insight into the interactions between patient and machine on overnight studies. Active upper airway occlusion, probably at the level of the glottis, has been proposed as a cause of the failure of noninvasive assisted ventilation [19]. In another more recent paper, the initial peak in mask pressure produced by the Monnal D is given as evidence of obstruction to airflow [14]. We have shown that this early peak is due to the supplementation of flow from the reservoir bag, and is present with even a moderate airway resistance of $5 \mathrm{cmH}_{2} \mathrm{O} \cdot \mathrm{L}^{-1} \cdot \mathrm{s}$ and cannot, therefore, be taken in isolation as evidence for obstruction.

\section{The effect of a leak in the patient circuit}

The results of introducing a leak into the circuit are shown in figure 4 . The $V \mathrm{~T}$ of the volume preset ventilators, delivered to the lung simulator, fell by $>50 \%$

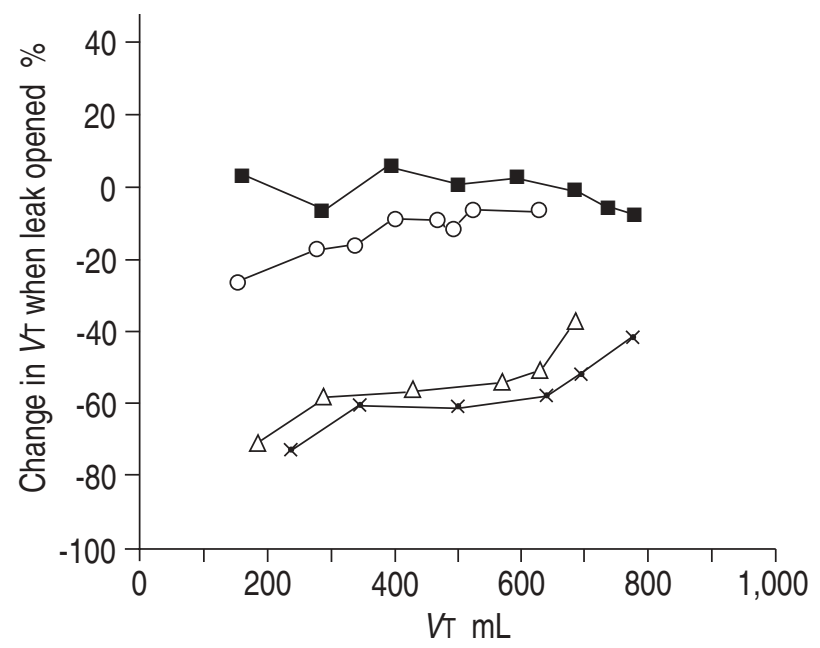

Fig. 4. - Effect of an additional leak on tidal volume. The realtionship between tidal volume $(V \mathrm{~T})$ with leak opened (ordinate) and $V \mathrm{~T}$ of the four distinct brands of preset ventilators. $\triangle \triangle$ : Monnal D (Taema); — — : Nippy (Friday Medical); $\longrightarrow$ - Companion 2801 (Puritan Bennett); — : Bilevel Positive Airway Pressure (BiPAP; Respironics). over most of the range of preset volumes. This was associated with a fall in PAP of a similar magnitude. The pressure preset ventilators compensated well for the leak, with the BiPAP performing particularly well. Leaks around the patient interface or from the mouth are common during NiIPPV, especially with a nasal mask, and may lead to a failure of assisted ventilation [9]. The ability to compensate for leaks, as demonstrated by the BiPAP and the Nippy, might improve ventilatory support under such circumstances. Whatever the cause of a leak, it has been shown that recovery from arterial desaturation is often associated with arousal and increased patient effort [14], and the response of the ventilator to patient effort might be equally important.

\section{The effects of patient effort}

The minute volumes produced by each of the ventilators are plotted against the duration of the negative pressure in the box in figure 5. Two points on the graph are of particular interest. When the duration of the negative pressure in the box is $0.25 \mathrm{~s}$, this models the situation where the patient triggers the positive pressure pump but there is no subsequent patient effort during the inspiratory phase. When the negative pressure in the box lasts for $1.5 \mathrm{~s}$, there is an almost complete overlap between the duration of patient effort and ventilator $t \mathrm{I}$ [10].

When the patient makes an inspiratory effort of sufficient duration to just trigger the ventilator, it would be expected that the resulting breath should have the same $V \mathrm{~T}$ as an untriggered breath for the Companion 2801 and the Nippy [10]. The minute volume should, therefore, increase in direct proportion to the rise in $f$ R, i.e. $33 \%$ in this test. The behaviour of the BiPAP is difficult to predict, since the change in the initial inspiratory flow caused by the trigger stimulus affects $t \mathrm{I}$.

The Companion 2801 behaved as expected. The slight excess in minute volume over that predicted was explained

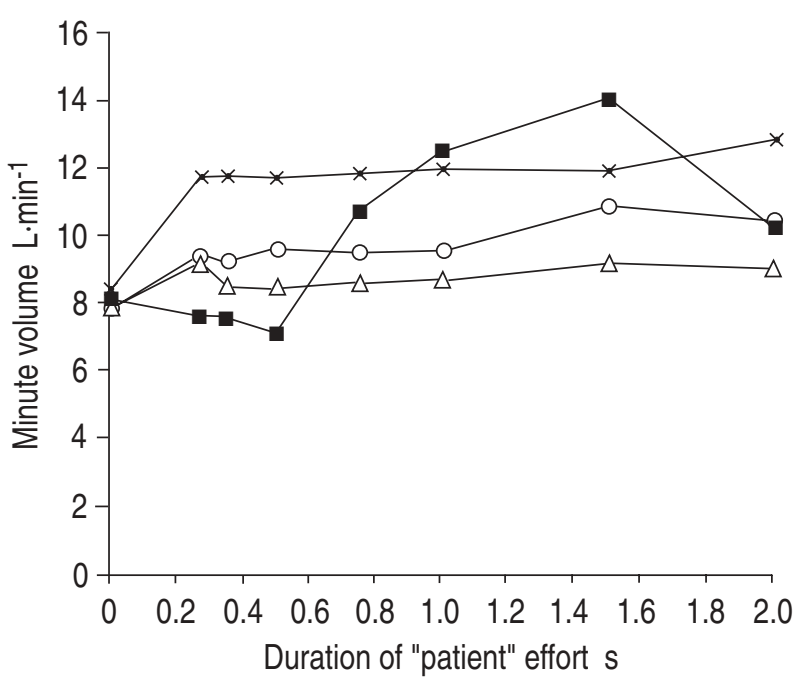

Fig. 5. - Effect of "patient" effort duration on minute volume. The minute volumes of the four distinct brands of ventilator plotted against the duration of negative pressure in the box. $\triangle \triangle$ : Monnal D (Taema); ——_ : Nippy (Friday Medical); ——: Companion 2801 (Puritan Bennett); —— Bilevel Positive Airway Pressure (BiPAP; Respironics). 
by the small volume change generated in the lung simulator by the negative pressure pump before the Companion 2801 was triggered. The Nippy did not behave as expected, with a rise in minute volume of only $17 \%$. This may, in part, be explained by a weakness of the model. The Negavent negative pressure pump produced back flow into the box at the start of its expiratory phase, and this led to a short positive pressure pulse before a return to atmospheric pressure in the box. When this was superimposed on the inspiratory phase of the Nippy, it led to a reduction in inspiratory flow into the lung simulator while airway pressure followed the predetermined waveform without perturbation.

The minute volume of the BiPAP fell by $7 \%$ when a brief patient effort was used to try to raise the ventilator rate. This fall is explained by a prolongation of $t \mathrm{I}$ for the triggered breaths, which produced two deleterious effects (fig. 6). Prolongation of $t \mathrm{I}$ led to an equivalent shortening of the expiratory time $(t \mathrm{E})$, which in turn produced progressive hyperinflation of the lung simulator, with decreasing $V \mathrm{~T}$ over a cycle of four breaths. On the fifth ventilator breath, again because of the prolongation of $t \mathrm{I}$, two of the negative pressure pulses fell on periods refractory to triggering. Thus, there was no overall increase in $f \mathrm{R}$ and some of the ventilator breaths had a smaller $V \mathrm{~T}$ compared to the nontriggered breaths. This pattern was stable over the $10 \mathrm{~min}$ of the test.

Similar episodes of desynchronization, associated with hyperinflation of the lungs, have been demonstrated in intubated patients on pressure support ventilation [20]. However, the ventilator employed was pressure-triggered and many of the episodes of desynchronization were thought to be due to elevated intrinsic positive end-expiratory pressure (PEEPi) which prevented the patients inspiratory effort from reducing airway pressure to the trigger level. We are not aware of previous reports of the failure of synchronization due to the progressive prolongation of $t \mathrm{I}$ which we have described for the flowtriggered BiPAP, but would suggest that in patients with airflow limitation liable to hyperinflation this might be a limitation of the BiPAP.

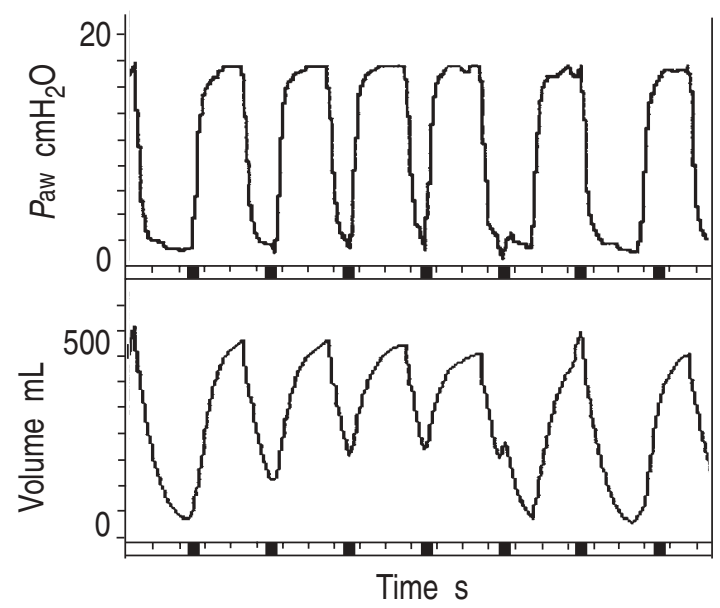

Fig. 6. - Intermittent triggering of the Bilevel Positive Airway Pressure (BiPAP; Respironics). Paw: airway pressure; Volume: volume in the lung stimulator. Trigger stimuli are marked as on the time axis. Triggers 2, 3 and 4 led to progressive hyperinflation and subsequent desynchronization, with the failure of triggers 5, 6 and 7 to capture the ventilator.
When the rate of the Monnal D is increased by triggering, the minute volume, which is preset, would be predicted to remain constant while $V \mathrm{~T}$ is reduced. In fact, the minute volume increased by $18 \%$. This may be partly explained by the additional inspiratory volume generated by the negative pressure pump, but was also due in part to an extra contribution from the reservoir bag. While the compressor in the Monnal D generates a constant flow, the bag delivers flow at a rate dependent on the pressure difference between itself and the airway. For a triggered breath, the airway pressure at the initiation of inspiration is negative; the pressure difference between the bag and the airway is greater than for a nontriggered breath and, therefore, the flow from the bag is higher at the start of inspiration giving a greater final $V \mathrm{~T}$. This mechanism can only operate when $t \mathrm{E}$ is of sufficient duration to allow the refilling of the bag.

When the patient effort is prolonged beyond that required to trigger the ventilator, no further increase in volume would be expected from a volume preset ventilator. This is in effect wasted effort for the patient. The Companion 2801 and the Monnal D both behaved as expected. For a pressure preset ventilator, the effective inspiratory driving pressure is the pressure difference across the lungs, i.e. the difference between the airway pressure and the pleural pressure [10] (or box pressure in the present model). For triggering plus prolonged patient effort, therefore, the minute volume would be expected to increase due both to the increase in $f^{\prime \prime} \mathrm{R}$ and to an increase in $V \mathrm{~T}$ secondary to this rise in effective driving pressure.

The minute volume for the Nippy did increase to a level slightly greater than expected from the increase in rate alone (38\% compared to $33 \%$ ). However, this was not to the level predicted from the calculated effective peak driving pressure of $22.27-(-8)=30.27 \mathrm{cmH}_{2} \mathrm{O}$, which from figure 2 would give a predicted increase in minute volume of $60 \%$. This shortfall is explained partly by a drop in the MAP and partly by a raised endexpiratory volume, due to the resistance of the expiratory valve of in the Nippy circuit. The BiPAP was triggered reliably when the negative pressure in the box was of similar duration to the BiPAP $t$ I. The increase in minute volume was similar to that expected from the compound effect of an increase in rate and an increase in the effective driving pressure.

In these investigations, we have used a patient simulator in order to measure the performance of each ventilator under reproducible conditions. This reproducibility itself makes the model an imperfect representation of the interaction between the ventilator and a patient, which may change from breath to breath. In addition, it might be expected that assisted ventilation alters the respiratory control in patients which is not reflected in the model. We have, however, shown that the ventilators tested produce distinct pressure waves, which may affect patient tolerance of the treatment, and that they respond differently to leaks and patient effort, which may influence the effectiveness of ventilatory support. These results show that in order to make informed decisions when choosing a ventilator for a particular patient and interpreting the results of clinical investigations using simple measures, such as the mask pressure, it is necessary to 
understand how the ventilators actually perform, and not to depend on the manufacturers description of the ventilator or extrapolations from theoretical models of how they should behave.

\section{References}

1. Ellis ER, Bye PTP, Bruderer JW, Sullivan CE. Treatment of respiratory failure during sleep in patients with neuromuscular disease. Am Rev Respir Dis 1987; 135: 148-152.

2. Ellis ER, Grunstein RR, Chan S, Bye PTP, Sullivan CE. Noninvasive ventilatory support during sleep improves respiratory failure in kyphoscoliosis. Chest 1988; 94 : 811-815.

3. Jackson M, Smith IE, King MA, Shneerson JM. Longterm noninvasive domiciliary assisted ventilation for respiratory failure following thoracoplasty. Thorax 1994; 49: 915-919.

4. Elliott MW, Simonds AK, Carroll MP, Wedzicha JA, Branthwaite MA. Domiciliary nocturnal nasal intermittent positive pressure ventilation in hypercapnic respiratory failure due to chronic obstructive lung disease: effects on sleep and quality of life. Thorax 1992; 47: 342-348.

5. Elliott MW, Steven MH, Phillips GD, Branthwaite MA. Noninvasive mechanical ventilation for acute respiratory failure. BMJ 1990; 300: 358-360.

6. Udwadia ZF, Santis GK, Steven MH, Simonds AK. Nasal ventilation to facilitate weaning in patients with chronic respiratory insufficiency. Thorax 1992; 47: 715-718.

7. Smith IE, Shneerson JM. A progressive care programme for prolonged ventilatory failure: analysis of outcomes. Br J Anaesth 1995; 75: 399-404.

8. Leger P, Bedicam JM, Cornette A, et al. Nasal intermittent positive pressure ventilation: long-term follow-up on patients with severe chronic respiratory insufficiency. Chest 1994;105: 100-105.

9. Bach JR, Alba AS. Management of chronic alveolar hypoventilation by nasal ventilation. Chest 1990; 97: 52-57.

10. Younes M. Proportional assist ventilation and pressure support ventilation: similarities and differences. Intensive Care Emerg Med 1991; 15: 361-380.

11. Meecham Jones DJ, Wedzicha JA. Comparison of pressure preset and volume preset nasal ventilator systems in stable chronic respiratory failure. Eur Respir J 1993; 6: 1060-1064.

12. Restrick LJ, Fox NC, Braid G, Ward EM, Paul EA, Wedzicha JA. Comparison of nasal pressure support ventilation with nasal intermittent positive pressure ventilation in patients with nocturnal hypoventilation. Eur Respir J 1993; 6: 364-370.

13. Elliott MW, Aquilina R, Green M, Moxham J, Simonds AK. A comparison of different modes of noninvasive ventilatory support: effects on ventilation and inspiratory muscle effort. Anaesthesia 1994; 49: 279-283.

14. Bach JR, Robert D, Leger P, Langevia B. Sleep fragmentation in kyphoscoliotic individuals with alveolar hypoventilation treated with NIPPV. Chest 1995; 107: 1552-1558.

15. Sanders MH, Kern N. Obstructive sleep apnea treated by independently adjusted inspiratory and expiratory positive airway pressure via nasal mask. Chest 1990; 98: 317-324.

16. Smith IE, Shneerson JM. Choosing a negative pressure ventilator pump: are there any important differences? Eur Respir J 1995; 8: 1792-1795.

17. Foglio K, Clini E, Vitacca M. Different modes of noninvasive intermittent positive pressure ventilation in acute exacerbations of chronic obstructive lung disease patients. Monaldi Arch Chest Dis 1994; 49: 556-557.

18. Sheehan GJ, Miedzinski LJ, Schroeder DG. Pneumothorax complicating BiPAP treatment for Pneumocystis carinii pneumonia. Chest 1993; 103: 1310.

19. Delguste P, Aubert Tulkens G, Rodenstein DO. Upper airway obstruction during nasal intermittent positive pressure hyperventilation in sleep. Lancet 1991; 338: 1295-1297.

20. Fabry B, Guttmann J, Eberhard L, Bauer T, Haberthur C, Wolff G. An analysis of desynchronization between the spontaneously breathing patient and ventilator during inspiratory pressure support. Chest 1995;107: 1387-1394. 